\title{
On $k$-Ordered Bipartite Graphs
}

\author{
Jill R. Faudree \\ University of Alaska Fairbanks \\ Fairbanks, AK 99775 \\ ffjrf@aurora.uaf.edu \\ Florian Pfender \\ Emory University \\ Atlanta, GA 30322 \\ fpfende@mathcs.emory.edu
}

\author{
Ronald J. Gould \\ Emory University \\ Atlanta, GA 30322 \\ rg@mathcs . emory. edu \\ Allison Wolf \\ College of Computing \\ Georgia Institute of Technology \\ Atlanta, GA 30332 \\ awolf@cc.gatech.edu
}

Submitted: Oct 30, 2001; Accepted: Mar 26, 2003; Published: Apr 15, 2003

MSC Subject Classifications: 05C35, 05C45

\begin{abstract}
In 1997, Ng and Schultz introduced the idea of cycle orderability. For a positive integer $k$, a graph $G$ is $k$-ordered if for every ordered sequence of $k$ vertices, there is a cycle that encounters the vertices of the sequence in the given order. If the cycle is also a hamiltonian cycle, then $G$ is said to be $k$-ordered hamiltonian. We give minimum degree conditions and sum of degree conditions for nonadjacent vertices that imply a balanced bipartite graph to be $k$-ordered hamiltonian. For example, let $G$ be a balanced bipartite graph on $2 n$ vertices, $n$ sufficiently large. We show that for any positive integer $k$, if the minimum degree of $G$ is at least $(2 n+k-1) / 4$, then $G$ is $k$-ordered hamiltonian.
\end{abstract}

\section{Introduction}

Over the years, hamiltonian graphs have been widely studied. A variety of related properties have also been considered. Some of the properties are weaker, for example traceability in graphs, while others are stronger, for example hamiltonian connectedness. Recently a new strong hamiltonian property was introduced in [3].

We say a graph $G$ on $n$ vertices, $n \geq 3$, is $k$-ordered for an integer $k, 1 \leq k \leq n$, if for every sequence $S=\left(x_{1}, x_{2}, \ldots, x_{k}\right)$ of $k$ distinct vertices in $G$ there exists a cycle that contains all the vertices of $S$ in the designated order. A graph is $k$-ordered hamiltonian if for every sequence $S$ of $k$ vertices there exists a hamiltonian cycle which encounters the vertices in $S$ in the designated order. We will always let $S=\left(x_{1}, x_{2}, \ldots, x_{k}\right)$ denote the ordered $k$-set. If we say a cycle $C$ contains $S$, we mean $C$ contains $S$ in the designated 
order under some orientation. The neighborhood of a vertex $v$ will be denoted by $N(v)$, the degree of $v$ by $d(v)$, the degree of $v$ to a subgraph $H$ by $d_{H}(v)$, and the minimum degree of a graph $G$ by $\delta(G)$. A graph on $n$ vertices is said to be $k$-linked if $n \geq 2 k$ and for every set $\left\{x_{1}, \ldots, x_{k}, y_{1}, \ldots, y_{k}\right\}$ of $2 k$ distinct vertices there are vertex disjoint paths $P_{1}, \ldots, P_{k}$ such that $P_{i}$ joins $x_{i}$ to $y_{i}$ for all $i \in\{1, \ldots, k\}$. Clearly, a $k$-linked graph is also $k$-ordered.

In the process of finding bounds implying a graph to be $k$-ordered hamiltonian, $\mathrm{Ng}$ and Schultz [3] showed the following:

Proposition 1. [3] Let $G$ be a hamiltonian graph on $n$ vertices, $n \geq 3$. If $G$ is $k$-ordered, $3 \leq k \leq n$, then $G$ is $(k-1)$-connected.

Theorem 2. [3] Let $G$ be a graph of order $n \geq 3$ and let $k$ be an integer with $3 \leq k \leq n$. If

$$
d(x)+d(y) \geq n+2 k-6
$$

for every pair $x, y$ of nonadjacent vertices of $G$, then $G$ is $k$-ordered hamiltonian.

Faudree et al.[4] improved the last bound as follows.

Theorem 3. [4] Let $G$ be a graph of sufficiently large order $n$. Let $k \geq 3$. If

$$
\delta(G) \geq \begin{cases}\frac{n+k-3}{2}, & \text { if } k \text { is odd } \\ \frac{n+k-2}{2}, & \text { if } k \text { is even },\end{cases}
$$

then $G$ is k-ordered hamiltonian.

Theorem 4. [4] Let $G$ be a graph of sufficiently large order $n$. Let $k \geq 3$. If for any two nonadjacent vertices $x$ and $y$,

$$
d(x)+d(y) \geq n+\frac{3 k-9}{2}
$$

then $G$ is k-ordered hamiltonian.

Theorem 5. [4] Let $k$ be an integer, $k \geq 2$. Let $G$ be a $(k+1)$-connected graph of sufficiently large order $n$. If

$$
|N(x) \cup N(y)| \geq \frac{n+k}{2}
$$

for all pairs of distinct vertices $x, y \in V(G)$, then $G$ is k-ordered hamiltonian.

Much like results for hamiltonicity, smaller bounds are possible if we restrict $G$ to be a balanced bipartite graph. In fact, we get the following results:

Theorem 6. Let $G(A \cup B, E)$ be a balanced bipartite graph of order $2 n \geq 618$. Let $3 \leq k \leq \frac{n}{103}$. If $\delta(G) \geq 4 k-1$ and for any two nonadjacent vertices $x \in A$ and $y \in B$, $d(x)+d(y) \geq n+\frac{k-1}{2}$, then $G$ is k-ordered hamiltonian. 
The bound on the degree sum is sharp, as will be shown later with an example. The upper bound on $k$ comes out of the proof, the correct bound should be a lot larger and possibly as large as $n / 4$.

Corollary 7. Let $G$ be a balanced bipartite graph of order $2 n \geq 618$. Let $3 \leq k \leq \frac{n}{103}$. If

$$
\delta(G) \geq \frac{2 n+k-1}{4}
$$

then $G$ is k-ordered hamiltonian.

Theorem 8. Let $G(A \cup B, E)$ be a balanced bipartite graph of order $2 n \geq 618$. Let $3 \leq k \leq$ $\min \left\{\frac{n}{103}, \frac{\sqrt{n}}{4}\right\}$. If for any two nonadjacent vertices $x \in A$ and $y \in B, d(x)+d(y) \geq n+k-2$, then $G$ is k-ordered hamiltonian.

The last bound is sharp, as the following graph $G$ shows:

Let the vertex set $V:=A_{1} \cup A_{2} \cup B_{1} \cup B_{2} \cup B_{3}$, with $\left|A_{1}\right|=\left|B_{1}\right|=k / 2,\left|B_{2}\right|=k-1$, $\left|A_{2}\right|=n-k / 2,\left|B_{3}\right|=n-3 k / 2+1$. Let the edge set consist of all edges between $A_{1}$ and $B_{1}$ minus a $k$-cycle, all edges between $A_{1}$ and $B_{2}$, and all edges between $A_{2}$ and the $B_{i}$ for $i \in\{1,2,3\}$. Then $G$ has minimum degree $\delta(G)=3 k / 2-3$, minimal degree sum $n+k-3$, and $G$ is not $k$-ordered, as there is no cycle containing the vertices of $A_{1} \cup B_{1}$ in the same order as the cycle whose edges were removed between $A_{1}$ and $B_{1}$. This example further suggests the following conjecture, strengthening Theorem 6 to a sharp result:

Conjecture 9. Let $G(A \cup B, E)$ be a balanced bipartite graph of order $2 n$. Let $k \geq 3$. If $\delta(G) \geq \frac{3 k-1}{2}-2$ and for any two nonadjacent vertices $x \in A$ and $y \in B, d(x)+d(y) \geq$ $n+\frac{k-1}{2}$, then $G$ is k-ordered hamiltonian.

In some of the proofs the following theorem of Bollobás and Thomason[1] comes in handy.

Theorem 10. [1] Every 22k-connected graph is k-linked.

\section{Proofs}

In this section we will prove Theorem 6 and Theorem 8.

From now on, $A$ and $B$ will always be the partite sets of the balanced bipartite graph $G$, and for a subgraph $H \subset G, H^{A}:=H \cap A$ and $H^{B}:=H \cap B$ will be its corresponding parts.

The following result and its corollary, which give sufficient conditions for $k$-ordered to imply $k$-ordered hamiltonian, will make the proofs easier.

Theorem 11. Let $k \geq 3$ and let $G(A \cup B, E)$ be a balanced bipartite, $k$-ordered graph of order $2 n$. If for every pair of nonadjacent vertices $x \in A$ and $y \in B$

$$
d(x)+d(y) \geq n+\frac{k-1}{2},
$$

then $G$ is k-ordered hamiltonian. 
Proof: Let $S=\left\{x_{1}, x_{2}, \cdots, x_{k}\right\}$ be an ordered subset of the vertices of $G$. Let $C$ be a cycle of maximum order $2 c$ containing all vertices of $S$ in appropriate order. Let $L:=G-C$. Notice that $L$ is balanced bipartite, since $C$ is. Let $l:=|L| / 2=\left|L^{A}\right|=\left|L^{B}\right|$.

Claim 1. Either $L$ is connected or $L$ consists of the union of two complete balanced bipartite graphs.

To prove the claim, it suffices to show that $d_{L}(u)+d_{L}(v) \geq l$ for all nonadjacent pairs $u \in L^{A}, v \in L^{B}$. Suppose the contrary, that is, there are two such vertices $u, v$ with $d_{L}(u)+d_{L}(v)<l$. Since $d(u)+d(v) \geq n+(k-1) / 2$, it follows that $d_{C}(u)+d_{C}(v) \geq$ $c+(k+1) / 2$. There are no common neighbors of $u$ and $v$ on $C$, hence there are at least $k+1$ edges on $C$ with both endvertices adjacent to $\{u, v\}$. Fix a direction on $C$. Say there are $r$ edges on $C$ directed from a $u$-neighbor to a $v$-neighbor, and $t$ edges from a $v$-neighbor to a $u$-neighbor. Without loss of generality, let $r \geq t$. On $C$, between any two of the $r \geq(k+1) / 2$ edges of that type, there have to be at least two vertices of $S$, else $C$ could be enlarged (see Figure 1). Thus $|S| \geq k+1$, a contradiction, which proves the claim.

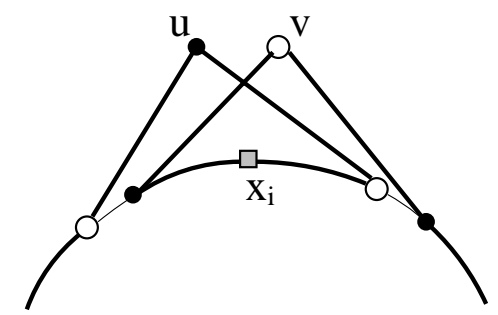

Figure 1:

In particular, the claim shows that there are no isolated vertices in $L$ and that all of $L$ 's components are balanced.

Suppose $l \geq 1$. Let $L_{1}$ be a component of $L, L_{2}:=L-L_{1}, l_{1}:=\left|L_{1}\right| / 2$, and $l_{2}:=\left|L_{2}\right| / 2$. The $k$ vertices of $S$ split the cycle $C$ into $k$ intervals: $\left[x_{1}, x_{2}\right],\left[x_{2}, x_{3}\right], \ldots$, $\left[x_{k}, x_{1}\right]$. Assume there are vertices $x, y \in L_{1}(x=y$ is possible $)$ with distinct neighbors in one of the intervals of $C$ determined by $S$, say $\left[x_{i}, x_{i+1}\right]$. Let $z_{1}$ and $z_{2}$ be the immediate successor and predecessor on $C$ to the neighbors of $x$ and $y$ respectively according to the orientation of $C$. Observe that we can choose $x$ and $y$ and their neighbors in $C$ such that none of the vertices on the interval $\left[z_{1}, z_{2}\right]$ have neighbors in $L_{1}$. We can also assume that $z_{1} \neq z_{2}$, otherwise $x=y$ by the maximality of $C$, and bypassing $z_{1}$ through $x$ would lead to a cycle of the same order, but the new outside component $L_{1}-x$ would not be balanced, a contradiction to claim 1 . Let $z$ be either $z_{2}$ or its immediate predecessor such that $z_{1}$ and $z$ are from different parts. Since $x$ and $y$ are in the same component of $L$, there is an $x, y$-path through $L$. Let $\bar{y}$ be either $y$ or its immediate predecessor on the path such that $x$ and $\bar{y}$ are from different parts. If $x=y$, let $\bar{y}$ be any neighbor of $x$ in $L$. Let $R$ be the path on $C$ from $z_{1}$ to $z_{2}$ and $r:=|R|$. Since $C$ is maximal, the $x, \bar{y}$-path 
can't be inserted, and since neither $x$ nor $\bar{y}$ have neighbors on $R$,

$$
d(x)+d(\bar{y}) \leq 2 l_{1}+\frac{2 c-r+1}{2} .
$$

Further, the $z_{1}$, z-path can't be inserted anywhere on $C-R$, else $C$ could be enlarged by inserting it and going through $L$ instead (or in the case $x=y$ we would get a same length cycle with unbalanced outside components). Since $z_{1}$ and $z$ have no neighbors in $L_{1}$, we get

$$
d\left(z_{1}\right)+d(z) \leq 2 l_{2}+r+\frac{2 c-r+1}{2} .
$$

Hence

$$
d(x)+d(\bar{y})+d\left(z_{1}\right)+d(z) \leq 2 l_{2}+2 l_{1}+2 c+1=2 n+1,
$$

which contradicts (with $k \geq 3$ ) that

$$
d(x)+d(z) \geq n+\frac{k-1}{2}
$$

and

$$
d(\bar{y})+d\left(z_{1}\right) \geq n+\frac{k-1}{2} .
$$

Thus, there is no interval $\left[x_{i}, x_{i+1}\right]$ with two independent edges to $L_{1}$. By Proposition 1 , $G$ is $(k-1)$-connected, thus all but possibly one of the segments $\left(x_{i}, x_{i+1}\right)$ have exactly one vertex with a neighbor in $L_{1}$.

Since $\left|N_{C}\left(L_{1}\right)\right| \leq k$, we assume without loss of generality that $\left|N_{C}\left(L_{1}^{B}\right)\right| \leq k / 2$. Let $x \in L_{1}^{B}$ and let $\left|N_{C}(x)\right|=d \leq k / 2$. Thus, for every $v \in C$ that is not adjacent to $L_{1}$ the degree sum condition implies:

$$
d(v) \geq n+\frac{k-1}{2}-\left(l_{1}+d\right)=c+l_{2}+\left(\frac{k}{2}-d-\frac{1}{2}\right) .
$$

On the other hand, we know $d(v) \leq c+l_{2}-1$. Thus, $d \geq 2$. Now we have shown that $N_{L_{1}}(C)$ includes vertices from both $L_{1}^{A}$ and $L_{1}^{B}$. So, without loss of generality, assume $L_{1}$ has neighbors $y$ and $z$ in $\left(x_{1} \ldots x_{2}\right)$ and $\left(x_{2} \ldots x_{3}\right)$ respectively and such that $y$ and $z$ are in different partite sets.

Let $y, z$ be the unique vertices in $\left(x_{1}, x_{2}\right)$ and $\left(x_{2}, x_{3}\right)$ respectively, which have neighbors in $L_{1}$. Since the successors of $y$ and $z$ are from different parts and not adjacent to $L_{1}$, they must be adjacent to each other. But now $C$ can be extended, which is a contradiction.

This proves that $L$ has to be empty. Therefore $C$ is hamiltonian.

An immediate Corollary to Theorem 11 is the following:

Corollary 12. Let $k \geq 3$ and let $G$ be a $k$-ordered balanced bipartite graph of order $2 n$. If $\delta(G) \geq \frac{n}{2}+\frac{k-1}{4}$, then $G$ is k-ordered hamiltonian. 
To see that these bounds are sharp, consider the following graph $G(A \cup B, E)$ :

$$
A:=A_{1} \cup A_{2}, B:=B_{1} \cup B_{2},
$$

with

$$
\begin{gathered}
\left|A_{1}\right|=\left|B_{1}\right|=\left\lceil\frac{n}{2}+\frac{k-1}{4}\right\rceil-1, \\
\left|A_{2}\right|=\left|B_{2}\right|=n-\left|A_{1}\right|,
\end{gathered}
$$

and

$$
E:=\left\{a b \mid a \in A_{1}, b \in B\right\} \cup\left\{a b \mid a \in A, b \in B_{1}\right\} .
$$

For $n$ sufficiently large, $G$ is obviously a $k$-connected, $k$-ordered, and balanced bipartite graph. The minimum degree is $\delta(G)=d(v)=\left|A_{1}\right|$ for any vertex $v \in B_{2} \cup A_{2}$, thus the minimum degree condition is just missed. But $G$ is not $k$-ordered hamiltonian, for if we consider $S=\left\{x_{1}, x_{2}, \ldots, x_{k}\right\},\left\{x_{1}, x_{3}, \ldots\right\} \subseteq A_{2},\left\{x_{2}, x_{4}, \ldots\right\} \subseteq B_{2}$. Let $C$ be a cycle that picks up $S$ in the designated order. Then $C \cap\left(A_{1} \cup B_{2}\right)$ consists of at least $\lfloor k / 2\rfloor$ paths, all of which start and end in $A_{1}$. Therefore $\left|C \cap A_{1}\right| \geq\left|C \cap B_{2}\right|+(k-1) / 2$. If $C$ was hamiltonian, it would follow that $\left|A_{1}\right| \geq\left|B_{2}\right|+(k-1) / 2$, which is not true.

The following easy lemmas will be useful.

Lemma 13. Let $G$ be a graph, let $k \geq 1$ be an integer and let $v \in V(G)$ with $d(v) \geq 2 k-1$ for some $k$. If $G-v$ is k-linked, then $G$ is k-linked.

Proof: This is an easy exercise.

Lemma 14. Let $G$ be a $2 k$-connected graph with a $k$-linked subgraph $H \subset G$. Then $G$ is $k$-linked.

Proof: Let $S:=\left\{x_{1}, \ldots, x_{k}, y_{1}, \ldots, y_{k}\right\}$ be a set of $2 k$ vertices in $G$, not necessarily disjoint from $H$. Since $G$ is $2 k$-connected, there are $2 k$ disjoint paths from $S$ to $H$, including the possibility of one-vertex paths. Since $H$ is $k$-linked, those paths can be joined in a way that $k$ paths arise which connect $x_{i}$ with $y_{i}$ for $1 \leq i \leq k$.

Lemma 15. Let $k \geq 1$. Let $G(A \cup B, E)$ be a bipartite graph with $d(v) \geq \frac{|B|}{2}+\frac{3 k}{2}$ for all $v \in A$, and $d(w) \geq 2 k$ for all $w \in B$. Then $G$ is $k$-linked.

Proof: Let $S:=\left\{x_{1}, \ldots, x_{k}, y_{1}, \ldots, y_{k}\right\}$ be a set of $2 k$ vertices in $G$. Pick a set $S^{\prime}:=\left\{x_{1}^{\prime}, \ldots, x_{k}^{\prime}, y_{1}^{\prime}, \ldots, y_{k}^{\prime}\right\} \subset A$ as follows: If $x_{i} \in A$ set $x_{i}^{\prime}=x_{i}$. Otherwise let $x_{i}^{\prime}$ be a neighbor of $x_{i}$ not in $S$. Similarly pick the $y_{i}^{\prime}$. It is possible to pick $2 k$ different vertices for $S^{\prime}$ since $d(w) \geq 2 k$ for all $w \in B$.

Now find disjoint paths of length 2 between $x_{i}^{\prime}$ and $y_{i}^{\prime}$ avoiding all the other vertices of $S$ for $1 \leq i \leq k$. This is possible since $\left|N\left(x_{i}^{\prime}\right) \cap N\left(y_{i}^{\prime}\right)\right| \geq d\left(x_{i}^{\prime}\right)+d\left(y_{i}^{\prime}\right)-|B| \geq 3 k$.

Proof of Theorem 6: By Theorem 11, it suffices to show that $G$ is $k$-ordered.

Let $K$ be a minimal cutset. If $|K| \geq 22 k$, then $G$ is $k$-linked by Theorem 10 . Therefore it is $k$-ordered. Assume now that $|K|<22 k$. We have to deal with two cases. 
Case 1. There is an isolated vertex $v \in G-K$.

Since $|K|=|N(v)| \geq \delta(G) \geq 4 k-1, G$ is $2 k$-connected, thus by Lemma 14 it suffices to find a $k$-linked subgraph. Without loss of generality, let $v \in B$. Let $R=G-K-v$. Then $d(w)>n-22 k$ for all $w \in R^{A}$. So there are at least $(n-22 k)^{2}$ edges in $R$, resulting in less than $23 k$ vertices $u \in R^{B}$ with $d_{R}(u)<2 k$. Let $H$ be the subgraph of $R$ induced by $R^{A}$ and the vertices of $R^{B}$ with $d_{R}(u) \geq 2 k$. For $w \in R^{A}$, we have $d_{H}(w) \geq n-45 k \geq \frac{\left|H^{B}\right|}{2}+\frac{3 k}{2}$, since $n>100 k$. By Lemma $15, H$ is $k$-linked.

Case 2. There are no isolated vertices in $G-K$.

First, observe that $G-K$ has exactly two components. Otherwise, for the three components $C_{1}, C_{2}, C_{3}$ choose vertices $v_{i} \in C_{i}^{A}, w_{i} \in C_{i}^{B}, 1 \leq i \leq 3$.

Then we can bound their degree sum as follows:

$$
\begin{aligned}
2 n+2|K| & \geq\left(\left|C_{1}\right|+|K|\right)+\left(\left|C_{2}\right|+|K|\right)+\left(\left|C_{3}\right|+|K|\right) \\
& \geq\left(d\left(v_{1}\right)+d\left(w_{1}\right)\right)+\left(d\left(v_{2}\right)+d\left(w_{2}\right)\right)+\left(d\left(v_{3}\right)+d\left(w_{3}\right)\right) \\
& =\left(d\left(v_{1}\right)+d\left(w_{2}\right)\right)+\left(d\left(v_{2}\right)+d\left(w_{3}\right)\right)+\left(d\left(v_{3}\right)+d\left(w_{1}\right)\right) \\
& \geq 3\left(n+\frac{k-1}{2}\right),
\end{aligned}
$$

a contradiction.

Call the two components $L$ and $R$. Without loss of generality, let $|R| \geq|L|$ and $\left|L^{A}\right| \geq\left|L^{B}\right|$. Let $v \in L^{A}, w \in L^{B}, x \in R^{A}, y \in R^{B}$. Then

$$
\begin{aligned}
& \left|L^{A}\right|+\left|R^{A}\right|+\left|K^{A}\right|=\left|L^{B}\right|+\left|R^{B}\right|+\left|K^{B}\right|=n, \\
& \left|L^{B}\right|+\left|R^{A}\right|+|K| \geq d(w)+d(x) \geq n+\frac{k-1}{2}, \\
& \left|L^{A}\right|+\left|R^{B}\right|+|K| \geq d(v)+d(y) \geq n+\frac{k-1}{2} .
\end{aligned}
$$

Thus, the inequalities above imply the parts of the components are of similar size:

$$
\begin{aligned}
& \left|L^{A}\right|-\left|L^{B}\right| \leq\left|K^{B}\right|-\frac{k-1}{2}, \\
& \left|R^{A}\right|-\left|R^{B}\right| \leq\left|K^{B}\right|-\frac{k-1}{2}, \\
& \left|R^{B}\right|-\left|R^{A}\right| \leq\left|K^{A}\right|-\frac{k-1}{2} .
\end{aligned}
$$

Further, we get the following bounds for the degrees inside the components:

$$
\begin{aligned}
d_{R}(y) & \geq n+\frac{k-1}{2}-d(v)-\left|K^{A}\right| \\
& \geq n+\frac{k-1}{2}-\left|L^{B}\right|-\left|K^{B}\right|-\left|K^{A}\right| \\
& =\left|R^{B}\right|-\left(\left|K^{A}\right|-\frac{k-1}{2}\right), \\
d_{R}(x) & \geq\left|R^{A}\right|-\left(\left|K^{B}\right|-\frac{k-1}{2}\right), \\
d_{L}(w) & \geq\left|L^{B}\right|-\left(\left|K^{A}\right|-\frac{k-1}{2}\right), \\
d_{L}(v) & \geq\left|L^{A}\right|-\left(\left|K^{B}\right|-\frac{k-1}{2}\right) .
\end{aligned}
$$


Claim 1. $R$ is k-linked.

By symmetry of the argument, we may assume that $\left|R^{B}\right| \geq\left|R^{A}\right|$, thus

$$
\left|R^{B}\right| \geq \frac{|R|}{2} \geq \frac{2 n-|K|-|L|}{2} \geq \frac{n}{2}-\frac{|K|}{4} .
$$

Now,

$$
\begin{aligned}
d_{R}(y) & \geq\left|R^{B}\right|-\left(\left|K^{A}\right|-\frac{k-1}{2}\right) \geq \frac{\left|R^{A}\right|}{2}+\frac{\left|R^{B}\right|}{2}-|K|+\frac{k-1}{2} \\
& \geq \frac{\left|R^{A}\right|}{2}+\frac{n}{4}-\frac{9|K|}{8}+\frac{k-1}{2} \geq \frac{\left|R^{A}\right|}{2}+\frac{103 k}{4}-\frac{9(22 k-1)}{8}+\frac{k-1}{2} \\
& >\frac{\left|R^{A}\right|}{2}+\frac{3 k}{2} .
\end{aligned}
$$

Further,

$$
d_{R}(x) \geq\left|R^{A}\right|-\left(\left|K^{B}\right|-\frac{k-1}{2}\right) \geq\left|R^{B}\right|-|K|+\frac{k-1}{2}>2 k .
$$

Hence, the conditions of Lemma 15 are satisfied for $R$, and $R$ is $k$-linked.

If $|K| \geq 2 k$, then $G$ is $k$-linked by Lemma 14 and we are done. So assume from now on $|K|<2 k$.

Claim 2. $L$ is k-linked.

If $|L|>n-2 k$, the proof is similar to the last case:

$$
d_{L}(v) \geq\left|L^{A}\right|-\left|K^{B}\right|+\frac{k-1}{2}>\frac{\left|L^{B}\right|}{2}+\frac{n-2 k}{4}-2 k+\frac{k-1}{2}>\frac{\left|L^{B}\right|}{2}+\frac{3 k}{2},
$$

and

$$
d_{L}(w) \geq\left|L^{A}\right|-\left(\left|K^{B}\right|-\frac{k-1}{2}\right)>\left|L^{B}\right|-|K|>2 k .
$$

Applying Lemma 15 to $L$ gives the result.

If $|L| \leq n-2 k, L$ is complete bipartite from the degree sum condition. Further, $\left|L^{A}\right| \geq\left|L^{B}\right| \geq d(v)-\left|K^{B}\right| \geq 2 k$ from the minimum degree condition, hence $L$ is $k$-linked. $\diamond$

Let $S:=\left\{x_{1}, x_{2}, \ldots, x_{k}\right\}$ be a set in $V(G)$. We want to find a cycle passing through $S$ in the prescribed order. Note that the minimum degree condition forces $|R| \geq|L| \geq|K|$. Assume $|K|=\kappa(G)=k+t$ where $t \geq-1$. Using the fact that $K$ is a minimal cut set, by Hall's Theorem (see for instance [2]) there is a matching of $K$ into $L$ and respectively $K$ into $R$, which together produce $k+t$ pairwise disjoint $P_{3}$ 's. Of all such matchings, pick one on either side with the fewest intersections with the set $S$.

Observe that a vertex $s \in K^{B}$ is either adjacent to every vertex of $L^{A}$ or $d(s)>n / 4$. Otherwise there would be a vertex $v \in L^{A}$ not connected to $s$, and $d(v)+d(s) \leq\left|L^{B}\right|+$ $\left|K^{B}\right|+n / 4 \leq n / 2-k+2 k+n / 4$, a contradiction. A similar argument shows that the analog statement is true for $s \in K^{A}$, since $\left|L^{A}\right|$ and $\left|L^{B}\right|$ differ by less than $|K|<2 k$. Hence, each vertex $s \in K$ has large degree to at least one of $L$ or $R$, in fact large enough that either $(L \cup\{s\})$ or $(R \cup\{s\})$ is $k$-linked. 
Assign every vertex of $K$ one by one to either $L$ or $R$ such that the new subgraphs $\bar{L}$ and $\bar{R}$ are still $k$-linked, applying Lemma 13 repeatedly. Left over from the $P_{3}$ 's is now one matching with $k+t$ edges between $\bar{L}$ and $\bar{R}$. We call an edge of this matching a double if both its endvertices are in $S$ and a single if exactly one endvertex is in $S$. If an edge is disjoint from $S$, we call it free.

We claim that the number of doubles is at most $t$ if $k$ is even and at most $t+1$ if $k$ is odd. Let $l^{A}$ (and respectively $r^{A}$ ) be the number of doubles which are edges between $L^{A}$ and $K^{B}$ (respectively between $R^{A}$ and $K^{B}$ ). Define $l^{B}$ and $r^{B}$ similarly. Note that this means $d:=l^{A}+l^{B}+r^{A}+r^{B}$ is the number of doubles. Let $v \in L^{A}-S, w \in L^{B}-S, x \in R^{A}-S$ and $y \in R^{B}-S$ such that none of those vertices are on an edge of the matching (this is possible since $\left|L^{A}\right|-\left|K^{B}\right| \geq 2 k,\left|L^{B}\right|-\left|K^{A}\right| \geq 2 k$ from the minimum degree condition). Then

$$
2 n+2\left\lceil\frac{k-1}{2}\right\rceil \leq d(v)+d(w)+d(x)+d(y) \leq 2 n+k+t-l^{A}-l^{B}-r^{A}-r^{B} .
$$

If $d \geq t+1$ for $k$ even or $t+2$ for $k$ odd, we obtain a contradiction to the above inequality.

Let $c$ be the number of elements of $S$ that are not vertices on any of the $k+t$ edges of the matching. Then $t+d+c$ of the edges are free. We are now prepared to construct the cycle containing the set $\left\{x_{1}, x_{2}, \cdots, x_{k}\right\}$ by constructing a set of disjoint $x_{i}, x_{i+1}$-paths, using that $\bar{L}$ and $\bar{R}$ are $k$-linked. Note that in constructing each $x_{i}, x_{i+1}$-path, using a free edge is only necessary if (1) $x_{i}$ is not on a single and (2) $x_{i}$ and $x_{i+1}$ are on different sides. If $k$ is even, these two conditions can occur at most $2 d+c$ times. If $k$ is odd, these two conditions can occur at most $2 d-1+c$ times (because of the parity, condition 2 cannot occur for every vertex). But neither ever exceeds $t+d+c$, the number of free edges. Hence, we may form a cycle containing the elements of $S$ in the appropriate order.

Proof of Theorem 8: By Theorem 11 it suffices to show that $G$ is $k$-ordered.

If the minimum degree $\delta(G) \geq 4 k-1$, then we are done by Theorem 6 . Thus, suppose that $s \in A$ is a vertex with $d(s)<4 k-1$. Let $R$ be the induced subgraph of $G$ on the following vertex set:

$$
\begin{gathered}
R^{B}:=\{v \in B: s v \notin E\}, \\
R^{A}:=\left\{w \in A: d_{R^{B}} \geq 2 k\right\} .
\end{gathered}
$$

The degree sum condition guarantees $d(v) \geq n-3 k$ for all $v \in R^{B}$. Further, $\left|R^{B}\right|=$ $n-d(s) \geq n-4 k+2$. It is easy to see that $\left|R^{A}\right|>n-4 k$ and that all the conditions for Lemma 15 are satisfied. Hence, $R$ is $k$-linked.

Let $H$ be the biggest $k$-linked subgraph of $G$. If $G=H$, we are done. Otherwise, let $L:=G-H$. The size of $L$ is $|L|=2 n-|H| \leq 2 n-|R| \leq 8 k$. Observe that no vertex $v \in L$ has $d_{H}(v)>2 k-2$, otherwise $V(H) \cup\{v\}$ would induce a bigger $k$-linked subgraph by Lemma 13 . Hence, no vertex in $L$ has degree greater than $10 k$, and therefore, $L$ is complete bipartite.

Define

$$
\alpha:=\min \left\{\left\{d_{H}(v) \mid v \in L^{A}\right\} \cup\{2 k\}\right\},
$$




$$
\beta:=\min \left\{\left\{d_{H}(v) \mid v \in L^{B}\right\} \cup\{2 k\}\right\} .
$$

Since $L$ is small, there are vertices $x \in H^{A}, y \in H^{B}$, with $N(x) \cup N(y) \subset H$. If $L^{A}=\emptyset$, then $\alpha=2 k$, and if $L^{B}=\emptyset$, then $\beta=2 k$. Either way, we get $\alpha+\beta \geq 2 k$.

Now assume that $L^{A} \neq \emptyset$ and $L^{B} \neq \emptyset$. Let $v \in L^{A}$ such that $d_{H}(v)=\alpha$. Then

$$
n+k-2 \leq d(v)+d(y) \leq d(v)+\left|H^{A}\right|=d(v)+n-\left|L^{A}\right| .
$$

Thus, $d(v) \geq\left|L^{A}\right|+k-2$, and

$$
\left|L^{B}\right|+\alpha=d(v) \geq\left|L^{A}\right|+k-2 .
$$

Analogously, let $w \in L^{B}$ with $d_{H}(w)=\beta$, then

$$
n+k-2 \leq d(w)+d(x) \leq d(w)+\left|H^{B}\right|=d(w)+n-\left|L^{B}\right|,
$$

and thus $d(w) \geq\left|L^{B}\right|+k-2$ and

$$
\left|L^{A}\right|+\beta=d(w) \geq\left|L^{B}\right|+k-2 .
$$

Therefore,

$$
\alpha+\beta \geq 2 k-4 \text {. }
$$

Let $S:=\left\{x_{1}, x_{2}, \ldots, x_{k}\right\}$ be a set in $V(G)$. From now on, all the indices are modulo $k$. To build the cycle, we need to find paths from $x_{i}$ to $x_{i+1}$ for all $1 \leq i \leq k$.

If $x_{i}$ and $x_{i+1}$ are neighbors, just use the connecting edge as path. Now, for all other $x_{i} \in L$ we find two neighbors $y_{i}$ and $z_{i}$ not in $S$. If $x_{i}$ and $x_{i+i}$ have a common neighbor $v$ which is not already used, set $z_{i}=y_{i+1}=v$. Afterwards, we can find distinct $y_{i}$ and $z_{i}$ by the following count: Suppose $x_{i} \in L^{A}$, so we need to find $y_{i}, z_{i} \in N\left(x_{i}\right)-U_{i}$, where

$$
U_{i}:=N\left(x_{i}\right) \cap\left\{\left\{x_{j}, y_{j}, z_{j}:|i-j|>1\right\} \cup\left\{z_{i+1}, y_{i-1}\right\}\right\} .
$$

For every $x_{j} \in L^{A},|i-j|>1$, there can be at most two vertices in $U_{i}$. For $x_{j} \in L^{A},|i-j|=$ 1 , there can be at most one vertex in $U_{i}$. For $x_{j} \in B,|i-j|>1$, there can be at most one vertex in $U_{i}$. Hence,

$$
\left|U_{i}\right| \leq 2\left|L^{A} \cap S-\left\{x_{i-1}, x_{i}, x_{i+1}\right\}\right|+2+\left|B \cap S-\left\{x_{i-1}, x_{i}, x_{i+1}\right\}\right| \leq\left|L^{A}\right|+k-4,
$$

and since $d\left(x_{i}\right) \geq\left|L^{A}\right|+k-2$, we can pick $y_{i}$ and $z_{i}$.

Try to choose as few $y_{i}, z_{i}$ out of $L$ as possible (i.e. pick as many as possible in $H$ ). Now for all $y_{i}, z_{j}$, where $y_{i} \neq z_{i-1}, z_{j} \neq y_{j+1}$, choose vertices $y_{i}^{\prime}, z_{i}^{\prime} \in H$ as follows: If $y_{i} \in H$, let $y_{i}^{\prime}=y_{i}$, if $z_{i} \in H$, let $z_{i}^{\prime}=z_{i}$. Otherwise, let $y_{i}^{\prime}$ be a neighbor of $y_{i}$ in $H$, and let $z_{i}^{\prime}$ be a neighbor of $z_{i}$ in $H$, which is not already used. We need to check if there is a vertex in $N\left(y_{i}\right) \cap H$ available.

Let $O_{i}=\left(N\left(x_{i}\right) \cup N\left(y_{i}\right)\right) \cap H$. We know that

$$
\left|O_{i}\right|=d_{H}\left(x_{i}\right)+d_{H}\left(y_{i}\right) \geq \alpha+\beta \geq 2 k-4 .
$$


For every $j \notin\{i-1, i, i+1\},\left|O_{i} \cap\left\{x_{j}, y_{j}, z_{j}, y_{j}^{\prime}, z_{j}^{\prime}\right\}\right| \leq 2$, and for $j=i+1, \mid O_{i} \cap$ $\left\{x_{j}, y_{j}, y_{j}^{\prime}\right\} \mid \leq 1$. This is a total count of at most $2 k-5$, at least one is left over for $y_{i}^{\prime}$. Observe that $y_{i}^{\prime} \notin N\left(x_{i}\right)$, otherwise we would have chosen it to be $y_{i}$, so in fact $y_{i}^{\prime} \in N\left(y_{i}\right)$. A similar count shows the availability of a vertex for $z_{i}^{\prime}$, with one possible exception: The one vertex left over could be $y_{i}^{\prime}$. This is only a problem if the count for $y_{i}^{\prime}$ gave us exactly one available vertex, otherwise we can just pick a different $y_{i}^{\prime}$. But now we can switch the vertices $y_{i}$ and $z_{i}$, and choose $y_{i}^{\prime}$ from $\left\{x_{i+1}, y_{i+1}, y_{i+1}^{\prime}\right\}$ (one of those is in $N\left(x_{i}\right) \cup N\left(y_{i}\right)$, since the count of used vertices gave exactly $2 k-5)$, and choose $z_{i}^{\prime}$ from $\left\{x_{i-1}, y_{i-1}, y_{i-1}^{\prime}\right\}$.

For all $x_{i} \in H$, set $y_{i}^{\prime}=z_{i}^{\prime}=x_{i}$. Since $H$ is $k$-linked, we can now find $z_{i}^{\prime}, y_{i+1}^{\prime}$-paths inside $H$ for all needed indices to complete the cycle.

\section{$3 \quad$ Further Results}

We also looked at the following closely related property:

Definition 1. We say a graph $G$ is k-ordered connected if for every sequence $S=$ $\left(x_{1}, x_{2}, \ldots, x_{k}\right)$ of $k$ distinct vertices in $G$, there exists a path from $x_{1}$ to $x_{k}$ that contains all the vertices of $S$ in the given order. A graph is k-ordered hamiltonian connected if there is always a hamiltonian path from $x_{1}$ to $x_{k}$ which encounters $S$ in the designated order.

Along the lines of the proofs in [4], you can show the following theorems for this property:

Theorem 16. Let $G$ be a graph of sufficiently large order $n$. Let $k \geq 3$. If

$$
\delta(G) \geq \frac{n+k-3}{2},
$$

then $G$ is k-ordered hamiltonian connected.

Theorem 17. Let $G$ be a graph of sufficiently large order $n$. Let $k \geq 3$. If for any two nonadjacent vertices $x$ and $y, d(x)+d(y) \geq n+\frac{3 k-6}{2}$, then $G$ is $k$-ordered hamiltonian connected.

The proofs do not give any new insights, so we will not present them here.

\section{References}

[1] B. Bollobás, C. Thomason, Highly Linked Graphs, Combinatorica 16 (1996), no.3, 313-320.

[2] G. Chartrand, L. Lesniak, "Graphs \& Digraphs", Chapman and Hall, London, 1996.

[3] L. Ng, M. Schultz, k-Ordered Hamiltonian Graphs, J. Graph Theory 1 (1997), 45-57. 
[4] J.Faudree, R.Faudree, R.Gould, M.Jacobson, L.Lesniak, On k-Ordered Graphs, J. Graph Theory 35 (2000), no.2, 69-82. 